\title{
Three-wave interaction in two-component quadratic nonlinear lattices
}

\author{
Konotop, V. V.; Cunha, M. D.; Christiansen, Peter Leth; Clausen, Carl A. Balslev
}

Published in:

Physical Review E. Statistical, Nonlinear, and Soft Matter Physics

Link to article, DOI:

10.1103/PhysRevE.60.6104

Publication date:

1999

Document Version

Publisher's PDF, also known as Version of record

Link back to DTU Orbit

Citation (APA):

Konotop, V. V., Cunha, M. D., Christiansen, P. L., \& Clausen, C. A. B. (1999). Three-wave interaction in twocomponent quadratic nonlinear lattices. Physical Review E. Statistical, Nonlinear, and Soft Matter Physics, 60(5), 6104-6110. https://doi.org/10.1103/PhysRevE.60.6104

\section{General rights}

Copyright and moral rights for the publications made accessible in the public portal are retained by the authors and/or other copyright owners and it is a condition of accessing publications that users recognise and abide by the legal requirements associated with these rights.

- Users may download and print one copy of any publication from the public portal for the purpose of private study or research.

- You may not further distribute the material or use it for any profit-making activity or commercial gain

- You may freely distribute the URL identifying the publication in the public portal 


\title{
Three-wave interaction in two-component quadratic nonlinear lattices
}

\author{
V. V. Konotop and M. D. Cunha \\ Department of Physics and Center of Mathematical Sciences, University of Madeira, 9000 Funchal, Portugal \\ P. L. Christiansen and C. Balslev Clausen \\ Department of Mathematical Modelling, Technical University of Denmark, DK-2800 Lyngby, Denmark
}

(Received 9 February 1999)

\begin{abstract}
We investigate a two-component lattice with a quadratic nonlinearity and find with the multiple scale technique that integrable three-wave interaction takes place between plane wave solutions when these fulfill resonance conditions. We demonstrate that energy conversion and pulse propagation known from three-wave interaction is reproduced in the lattice and that exact phase matching of parametric processes can be obtained in non-phase-matched lattices by tilting the interacting plane waves with respect to each other.
\end{abstract}

[S1063-651X(99)15110-9]

PACS number(s): 45.30.+s, 42.65.Ky, 42.82.Et, 04.30.Nk

\section{INTRODUCTION}

The diverse variety of research results from recent years has demonstrated that multiple-wave parametric processes contain a vast multitude of nonlinear phenomena. Recently, the study of parametric interaction has been extended to include discrete systems as well [3-10]. Here we will consider a two-component lattice formed by a line of resonantly interacting pairs of nonlinear oscillators. Most theoretical and experimental studies of parametric wave interactions are performed in the context of optics, but they are universal and may appear in all branches of nature where nonlinearity comes into play. Examples are found, e.g., in plasma physics, solid state physics, and in dynamics of electromagnetic, acoustic, and water waves $[1,2]$. We will nevertheless use the language of optics and understand the oscillators as an array of coupled waveguides, each of them allowing degenerate interaction between a fundamental and a second harmonic field. The evolution of the fields in the $n$th waveguide is governed by the following set of ordinary differentialdifference equations $[6,7]$ :

$$
\begin{gathered}
i \dot{W}_{n}+\left(W_{n+1}+W_{n-1}\right)+W_{n}^{*} V_{n}=0, \\
i \dot{V}_{n}+\eta\left(V_{n+1}+V_{n-1}\right)-\alpha V_{n}+W_{n}^{2} / 2=0,
\end{gathered}
$$

with $n=1, \ldots, n_{0} . W_{n}$ is the fundamental mode at frequency $\Omega_{1}$ that propagates inside the waveguide with wave vector $Q_{1}$ and $V_{n}$ is the second harmonic mode at frequency $\Omega_{2}$ $=2 \Omega_{1}$, which propagates with wave vector $Q_{2}$. System (1) is derived under the assumption that the modes are close to phase matching, i.e., $Q_{2} \approx 2 Q_{1}$. The constant $\alpha$ measures the residual phase mismatch between the modes. The positive constant $\eta=\kappa_{2} / \kappa_{1}$ is the ratio of the nearest neighbor coupling strength of the second harmonic $\kappa_{2}$ to the fundamental $\kappa_{1}$. It is thus assumed that $\kappa_{1} \neq 0$, i.e., that the fundamental fields in adjacent waveguides are overlapping. The dot denotes differentiation with respect to independent variable $z$, which measures the normalized distance along waveguides, and complex conjugation is indicated by an asterisk.
Various properties of the system (1) have already been established. In Ref. [5] all real, stationary solutions were found analytically for $n_{0}=2,4,6$, and in Ref. [6] the complete set of stationary solutions were found for $n_{0}=2$. When the number of sites is large, one can find several types of discrete solitary waves (also called intrinsic localized modes) with complicated phase and amplitude profiles [7,8]. Discrete solitary waves are intense and strongly localized excitations. This is in contrast to the plane wave solutions to Eqs. (1). In Ref. [10] the destiny of smooth nonlinear plane waves and the validity of modulation equations describing slow evolution of the solution parameters in the plane waves has been investigated in detail.

In this paper we will focus on interaction between three smoothly modulated (quasi-monochromatic) plane waves. Although the intensity of the plane waves varies smoothly, the fast phase variation will make a simple, direct continuum approximation of Eqs. (1) break down. Instead we use the multiple scale technique on the plane wave envelopes and find the well known three-wave interaction equations that are integrable and have soliton solutions [11]. We will explore the three-wave interaction equations as a means of generating interesting beam dynamics in the lattice. The paper is organized as follows. In Sec. II, modulation equations are derived. In Sec. III A, the degenerate case with only two participating waves is explored as a means by which to obtain phase-matched energy conversion to the second harmonic, using both plane waves and localized beams. In Sec. III B, we investigate the nondegenerate case with three plane waves of different frequency. We show that phase-matched down-conversion takes place via decay instability and explore the possibilities for beam steering in the lattice. Finally, Sec. IV contains a summary and conclusions.

\section{ENVELOPE EQUATIONS}

In the absence of coupling between the waveguides, the light fields in the fundamental and the second harmonic modes propagate steadily in the $z$ direction inside each isolated waveguide with wave vectors $Q_{1}$ and $Q_{2}$, respectively. However, the coupling to the neighboring waveguides results 


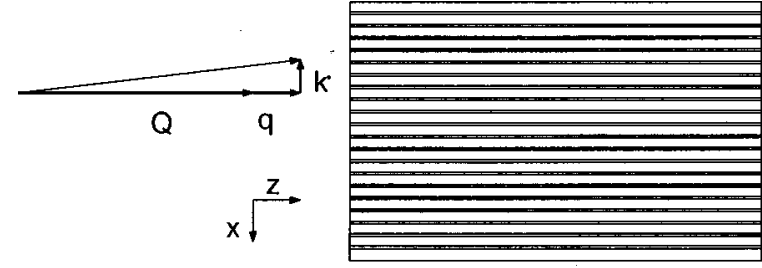

FIG. 1. Schematic view of an array of waveguides and wave vector of light beam(s).

in an effective lattice structure and leads to new modulation phenomena developing in the transverse $x$ direction associated with the waveguide numbers $n$ [12]. This modulation has a longitudinal part $q$ (along $z$ ) and a transverse part $k$ (along $x$ ), such that the total wave vector inside the waveguides is a sum of three vectors $Q, q$, and $k$. (See Fig. 1.) As long as Eqs. (1) are obtained in the long-wavelength limit, one has that $|q|,|k| \ll Q$, hence the oblique angles of the injected beams are small. In the present paper, we deal with resonance phenomena associated with $(q, k)$. Since the carrier waves $(\Omega, Q)$ of the modes $W_{n}$ and $V_{n}$ already fulfill the primary resonance, the resonance associated with $(q, k)$ may be called secondary or transverse due to the participation of a nonzero $k$.

In order to explain the phenomenon we are dealing with, we note that Eqs. (1) can be viewed as two linear lattices ( $V$ and $W$ ) which are coupled in a nonlinear way. In the linear limit where the lattices are decoupled, they are characterized by the dispersion relations

$$
q=2 \cos (k)
$$

for the $W$ branch and

$$
q=2 \eta \cos (k)-\alpha
$$

for the $V$ branch.

Consider now the evolution of an excitation that consists of three modes, characterized by transverse wave numbers $k_{1,2,3}$ and longitudinal wave number variations $q_{1,2,3}$ related by

$$
q_{3}=q_{1}+q_{2}, \quad k_{3}=k_{1}+k_{2}+\kappa,
$$

where $\kappa=2 \pi n(n=0, \pm 1, \pm 2, \ldots)$ is a vector of the reciprocal lattice. Equation (4) implies resonant interaction among the modes, which we refer to as transverse resonance. Assuming that the modes $k_{1,2}$ and $k_{3}$ belong to the $W$ and $V$ branches, respectively, one finds from Eqs. (2) and (3) that conditions (4) are satisfied provided that the following relation between the transverse wave numbers $k_{1,2}$ is fulfilled:

$$
\eta \cos \left(k_{1}+k_{2}\right)-\cos \left(k_{1}\right)-\cos \left(k_{2}\right)-\frac{\alpha}{2}=0 .
$$

Let us make two assumptions: (i) the lattice is infinite [14], and (ii) field excitations are smooth enough compared to the distance between neighbor sites, which is normalized to unity in our case. Then we can look for the solution of the system (1) in the form

$$
\begin{gathered}
W_{n}=\epsilon\left[A_{1}(T, X) e^{i\left(q_{1} z-k_{1} n\right)}+A_{2}(T, X) e^{i\left(q_{2} z-k_{2} n\right)}\right]+O\left(\epsilon^{3}\right), \\
V_{n}=\epsilon A_{3}(T, X) e^{i\left(q_{3} z-k_{3} n\right)}+\epsilon^{2} B_{1}(T, X) e^{2 i\left(q_{1} z-k_{1} n\right)} \\
+\epsilon^{2} B_{2}(T, X) e^{2 i\left(q_{2} z-k_{2} n\right)}+O\left(\epsilon^{3}\right) .
\end{gathered}
$$

Here $T=\epsilon z$ and $X=\epsilon n$ are slow coordinates and, consequently, amplitudes $A_{j}$ and $B_{j}$ are slowly varying complex functions. The terms characterized by the amplitudes $A_{j}$ describe modes interacting in a resonant manner while the terms characterized by the amplitudes $B_{j}$ describe nonresonant generation of second harmonics (see below).

Taking into account Eqs. (2) and (3), one ensures that Eqs. (4), (6), and (7) satisfy Eqs. (1) in the first order of the small parameter $\epsilon$. In the second order of parameter $\epsilon$, the dynamical equations for the amplitudes $A_{j}$ are found to be the well known system describing resonant three-wave interaction

$$
\begin{aligned}
& \frac{\partial A_{1}}{\partial T}+\mathrm{v}_{1} \frac{\partial A_{1}}{\partial X}=i A_{2}^{*} A_{3}, \\
& \frac{\partial A_{2}}{\partial T}+\mathrm{v}_{2} \frac{\partial A_{2}}{\partial X}=i A_{1}^{*} A_{3}, \\
& \frac{\partial A_{3}}{\partial T}+\mathrm{v}_{3} \frac{\partial A_{3}}{\partial X}=i A_{1} A_{2} .
\end{aligned}
$$

Here, $\mathbf{v}_{1,2}=-2 \sin \left(k_{1,2}\right)$ is related to the propagation angle of the $W$ modes and $\mathrm{v}_{3}=-2 \eta \sin \left(k_{3}\right)$ is related to the propagation angle of the $V$ mode.

In the present work we are concerned with the evolution of field excitations against a plane wave background. The simplest solutions having energy localized in space read [13]

$$
\begin{gathered}
A_{1}=\sqrt{\alpha_{2} \alpha_{3}} \operatorname{sech}(\zeta), \quad A_{2}=\sqrt{-\alpha_{1} \alpha_{3}} \tanh (\zeta), \\
A_{3}=i \sqrt{-\alpha_{1} \alpha_{2}} \operatorname{sech}(\zeta), \\
A_{1}=\sqrt{\alpha_{2} \alpha_{3}} \operatorname{sech}(\zeta), \quad A_{2}=\sqrt{\alpha_{1} \alpha_{3}} \operatorname{sech}(\zeta), \\
A_{3}=i \sqrt{\alpha_{1} \alpha_{2}} \tanh (\zeta),
\end{gathered}
$$

where $\alpha_{j}=\mathrm{v}_{j}-\mathrm{v}(j=1,2,3)$ and we have considered the change of variables $\zeta=X-\mathrm{v} T, \tau=T$; the constant $\vee$ is related to the propagation angle of the coupled state. The solutions (9) and (10) both require some relations among the parameters. In the case of solution (9), we need to satisfy $\mathrm{v}_{2,3}<\mathrm{v}<\mathrm{v}_{1}$ or $\mathrm{v}_{1}<\mathrm{v}<\mathrm{v}_{2,3}$ and in the case of solution (10) we need to satisfy $\mathrm{v}_{1,2,3}<\mathrm{v}$ or $\mathrm{v}_{1,2,3}>\mathrm{v}$. The amplitudes $B_{j}$ are also found from the second order approximation in $\epsilon$. One obtains

$$
B_{1}=\frac{\alpha_{2} \alpha_{3}}{b_{1}} \operatorname{sech}^{2}(\zeta), \quad B_{2}=-\frac{\alpha_{1} \alpha_{3}}{b_{2}} \tanh ^{2}(\zeta)
$$

in the case of solution (9) and

$$
B_{j}=\frac{\alpha_{3-j} \alpha_{3}}{b_{j}} \operatorname{sech}^{2}(\zeta),
$$


for solution (10). Here $b_{j}=4 q_{j}-4 \eta \cos \left(2 k_{j}\right)+2 \alpha(j=1,2)$. It follows from these expressions that solutions (6) and (7) are consistent unless one of the denominators in (11) and (12) vanishes:

$$
4 \cos \left(k_{1,2}\right)-2 \eta \cos \left(2 k_{1,2}\right)+\alpha=0 .
$$

The fulfillment of Eq. (13) is a special case. It is nothing more than the matching conditions of degenerate three-wave interaction, i.e., second harmonic generation in system (1) [see Eqs. (2), (3)]:

$$
q_{3}=2 q_{1,2}, \quad k_{3}=2 k_{1,2}+\kappa .
$$

In this case $A_{1}=A_{2}$ and the system (8) degenerates; the equations governing the second-harmonic generation read

$$
\begin{gathered}
\frac{\partial A_{1}}{\partial T}+\mathrm{v}_{1} \frac{\partial A_{1}}{\partial X}=i A_{1}^{*} A_{3}, \\
\frac{\partial A_{3}}{\partial T}+\mathrm{v}_{3} \frac{\partial A_{3}}{\partial X}=i A_{1}^{2},
\end{gathered}
$$

where we have considered the conditions of the secondharmonic generation in the form $q_{3}=2 q_{1}, k_{3}=2 k_{1}+\kappa$, which is equivalent to having $b_{1}=0$.

We check the linear stability of solutions (9) and (10), using their behavior in the asymptotic zone with respect to $X$. To this end, we linearize Eq. (8) about the unperturbed solutions (9) or (10) by making the substitution $A_{j} \rightarrow A_{j}$ $+g_{j}(\zeta, \tau)$, where the functions $A_{j}$ are given by Eq. (9) or Eq. (10) and $g_{j}(\zeta, \tau) \ll A_{j}$. The linearized equation can be written down in the form

$$
i \frac{\partial g}{\partial \tau}=\mathcal{L} g
$$

where $g=\operatorname{col}\left(g_{1}, g_{2}, g_{3}, g_{1}^{*}, g_{2}^{*}, g_{3}^{*}\right), \mathcal{L}=-i \alpha \partial / \partial \zeta+M, \alpha$ $=\operatorname{diag}\left(\alpha_{1}, \alpha_{2}, \alpha_{3}, \alpha_{1}, \alpha_{2}, \alpha_{3}\right)$, and $M$ is a $6 \times 6$ matrix with the elements $\quad M_{13}=M_{31}^{*}=-M_{46}^{*}=-M_{64}=A_{2}^{*}, \quad M_{15}=M_{24}$ $=-M_{42}^{*}=-M_{51}^{*}=A_{3}, \quad M_{23}=M_{32}^{*}=-M_{56}^{*}=-M_{65}=A_{1}^{*}$, while the remaining elements are zero. Then, instability of the solution means the existence of complex eigenvalues of $\mathcal{L}$ (we denote them $\omega$ ). Considering the asymptotics of the eigenvalue problem $\mathcal{L} g=\omega g$ at $\zeta \rightarrow \pm \infty$, we get $g_{j}$ $\rightarrow G_{j} e^{i K x}$, with $G_{j}$ being a constant. It is then possible to ensure that for the solution $(9), \omega(K)$ is a real-valued function for all $K$. In other words, the continuous spectrum of the operator $\mathbf{L}$ consists of intervals of the real axis $(-\infty$, $\left.-\omega_{0}\right] \cup\left[\omega_{0}, \infty\right)$, where $\omega_{0}=-2 \alpha_{1} \alpha_{3} /\left|\alpha_{1}-\alpha_{3}\right|$ (remember that for the unperturbed solution at hand $\left.\alpha_{1} \alpha_{3}<0\right)$. This result ensures the stability of the background.

On the other hand, in the same limit, $|\zeta| \rightarrow \infty$, the equation for $g_{2}$ (and for $g_{2}^{*}$ ) is singled out and reads

$$
i \alpha_{2} \frac{\partial g_{2}}{\partial \zeta}=\omega g_{2} \text {. }
$$

Thus the decay of $g_{2}$ at $\pm \infty$, simultaneously, cannot be provided unless $\omega=0$. This value of the spectral parameter corresponds to the only discrete eigenvalue of the operator $\mathcal{L}$ which is given by $g_{j}=\partial A_{j} / \partial \zeta$.
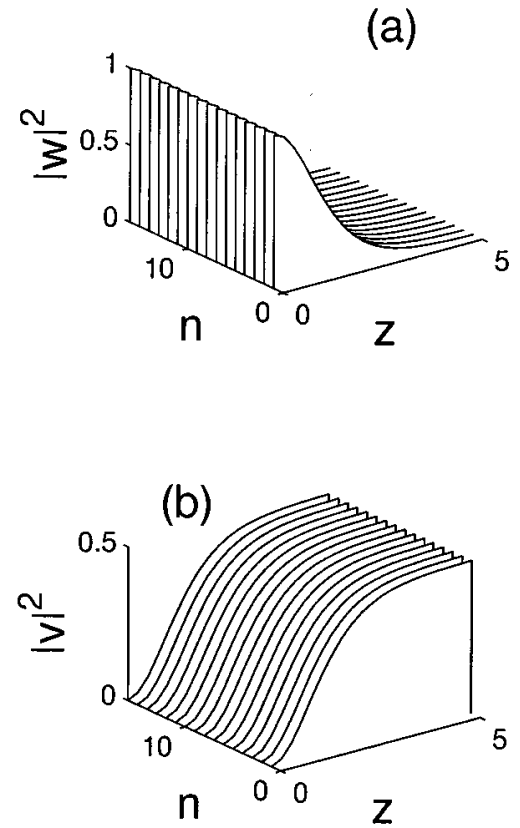

FIG. 2. Second harmonic generation with plane wave input. Parameters: $\alpha=-3, \eta=1$. The chain is periodic in order to simulate an infinite array.

In the case of solution (10), we find that $\omega$ has an imaginary part when $K^{2}<4 /\left(\mathrm{v}_{1}-\mathrm{v}_{3}\right)^{2}$ and hence even the background of the solution is unstable with respect to longwavelength excitations. This correlates with numerical simulations that will be presented in the next section. It should be mentioned here that the stability analysis provided above is in the context of the three-wave model and does not give the stability of the full discrete system of Eqs. (1).

\section{NUMERICAL RESULTS}

We have made a careful study based on numerical simulations of Eqs. (1). The purpose of the simulations is not only to check the validity of the approximate solutions from the preceding section. We also seek to visualize the beam dynamics in a comprehensive way and to investigate the effects that arise when parameter values are detuned and deviate from the optimal values. Recall that in Eqs. (1) and the solutions (9) and (10), $\eta$ and $\alpha$ are material parameters which are determined by the design and quality of the waveguides, while the wave numbers $k_{j}$ are free parameters and can be controlled by the proper choice of the input beam angles.

\section{A. Degenerate case}

First we present the results for the degenerate case in which we focus on the possibility of efficient energy conversion from the fundamental to the second harmonic. In the degenerate case, the resonance condition Eq. (13) is fulfilled and only two plane waves interact. Figure 2 gives an example of complete energy conversion from the fundamental to the second harmonic in an array with $n_{0}=16$ waveguides. Parameters are $\eta=1$ and $\alpha=-3$. At the input of the waveguides there is no second harmonic seed. It is seen that even though each waveguide has a nonzero phase mismatch it is possible to obtain complete energy conversion. This 


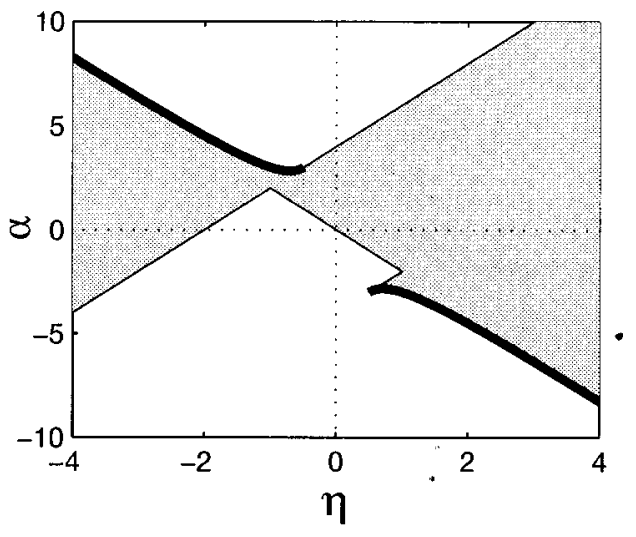

FIG. 3. Shaded area indicates the allowed parameter region for degenerate transverse phase matching. Thick lines indicate values with no walkoff, i.e., $v_{1}=v_{3}$.

would not be possible in a single isolated waveguide without a second harmonic seed with a well defined phase. However, Fig. 2 results from simulations with periodic boundary conditions. In this way the array is equivalent to an array of infinite extension with accuracy $1 / n_{0}$ [14]. A real array will have open boundary conditions unless a rather unrealistic circle geometry is assumed, or unless the array consists of only two waveguides, which makes periodic and open boundary conditions equivalent.

The resonance condition Eq. (13) cannot be satisfied for an arbitrary set of parameter values $\eta$ and $\alpha$. Figure 3 shows the region in parameter space where it is possible to choose the wave number $k=k_{1}$ in such a way that Eq. (13) is satisfied and degenerate transverse phase matching is obtained. The thick lines at the separating borders indicate the special values of $\eta$ and $\alpha$ where $\mathrm{v}_{1}=\mathrm{v}_{3}$ and the beams will propagate through the waveguides without walking away from each other in the transverse direction. The value $v_{3}-v_{1}$ is termed the walkoff. It becomes important when we consider the interaction of beams instead of plane waves of infinite extension.

With a plane wave as input, we had to impose the unnatural periodic boundary conditions. However, the boundary conditions are unimportant when the input condition is changed to the more realistic case with a confined beam. Figure 4 shows such a case. Equation (13) is fulfilled and $\mathrm{v}_{1}=\mathrm{v}_{3}$, so the fundamental beam is converted into a second harmonic beam that propagates in the same direction as the fundamental.

The values of $\eta$ and $\alpha$ are difficult to control or even determine exactly when an actual waveguide array is considered. Also, the input beam angle will have some uncertainty. Therefore, we consider the effect of tuning the parameter values away from the transverse phase-matching condition. Whether the value of $\eta, \alpha$, or $k$ is detuned, the qualitative effect remains the same. The detuning will manifest itself as an effective phase mismatch accompanied by a change in the walkoff. In order to monitor this, we have made a series of numerical simulations with the same configuration and input the beam shape as in Fig. 4 but with different values of $k$. Figure 5(a) plots the resulting energy conversion $\rho_{2 \omega}$ $=2|V|^{2} /|W|^{2}$ along the waveguides in four cases. In Fig. 5(b) we detect the bandwidth of the energy conversion process. It

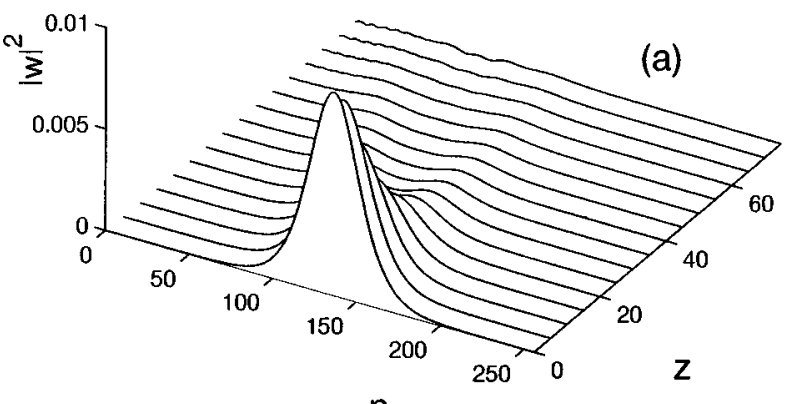

n

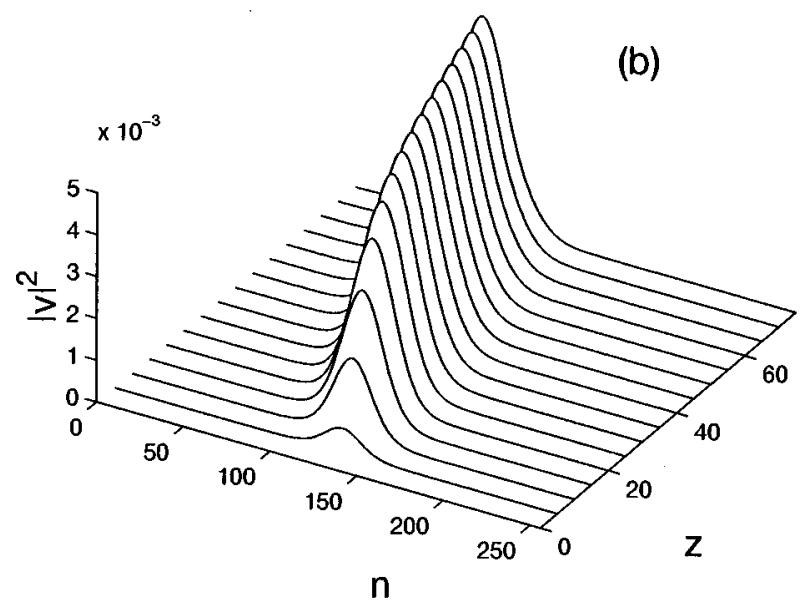

FIG. 4. Pulse generation under the transverse phase-matching condition. Parameters are $\eta=0.55, \alpha=-2.9$. Pulse parameters: $\epsilon=0.1$, width $=5$, velocity measured to $-0.8\left(\mathrm{v}_{1}=-0.83\right)$. (a) Fundamental. (b) Second harmonic.

is done in the following way. For each value of $k$ the energy conversion has a maximum somewhere along $z$. This value is recorded for a range of values of $k$ and is plotted in Fig. 5(b) for three different values of $\eta$.

\section{B. Three-wave interaction}

We now focus on genuine three-wave interaction for which Eq. (5) is fulfilled, while the condition for degenerate resonance Eq. (13) is not fulfilled. As a representative example of efficient three-wave interaction, Fig. 6 shows the process of down-conversion from one plane wave in $V$ to two plane waves in $W$ via decay instability in a lattice with 64 waveguides. The parameters for the sample are $\eta=0.5$ and $\alpha=-2.9$. At the entrance of the waveguides, a strong oblique plane wave with wave number $k_{3}=-0.25$ is input in the $V$ mode and a seed of vanishingly small amplitude with wave number $k_{1}=0.15$ is input in the $W$ mode. As seen in Fig. 6(a), the $k_{1}$ and the $k_{2}$ components build up in the $W$ mode. The presence of two plane waves in $W$ at $z=10$ generates an interference pattern in $|W|$ across the waveguides. The spectrum of $W$ yields that most of the energy of $W$ is in the $k_{1}$ and $k_{2}$ modes and a smaller amount of energy is in the sum and difference modes resulting from higher order mixing of the plane waves. 

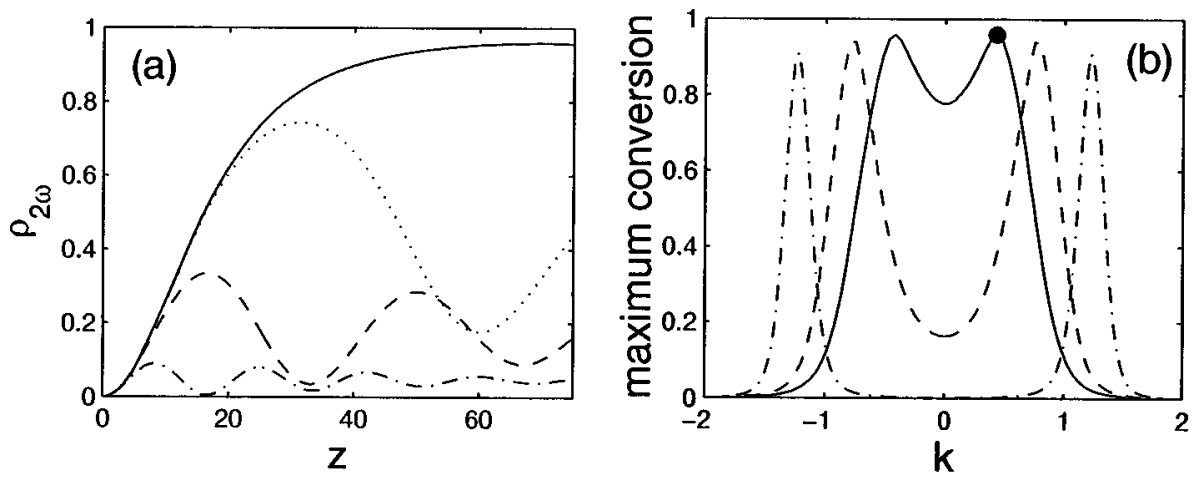

FIG. 5. Angle sensitivity in the second harmonic generation experiment. (a) Energy conversion $\rho_{2 \omega}$ versus $z$. Matching at $\pm k=0.43$ $\equiv k_{m}$ (solid line), detuned at $\pm k=k_{m}+0.2$ (dotted), $\pm k=k_{m}+0.4$ (dashed), and $\pm k=k_{m}+0.6$ (dash-dotted). (b) Peak conversion versus $k$ for various values of $\eta$. Solid line, $\eta=0.55$; dashed line, $\eta=0.7$; dash-dotted line, $\eta=1.5$. The big dot indicates the parameters of the experiment in Fig. 4.

We have repeated the experiment from Fig. 6, replacing the plane waves with beams localized to a limited number of waveguides. Also, the seed in $W$ is arbitrarily chosen to be $k_{2}$ instead of $k_{1}$. For the particular values of the wave numbers, the velocities are $\mathrm{v}_{1}=-0.2989, \mathrm{v}_{2}=0.7788$, and $\mathrm{v}_{3}$ $=0.2474$. The beams will therefore tend to walk away from

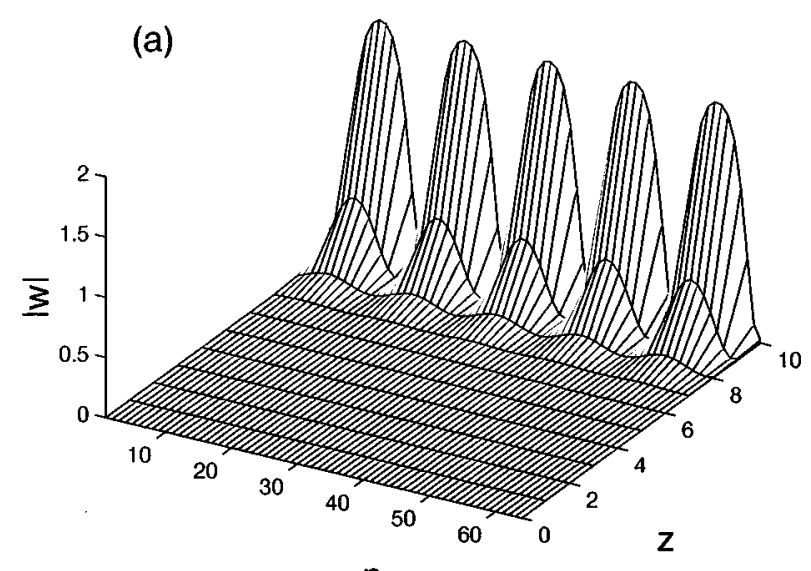

n

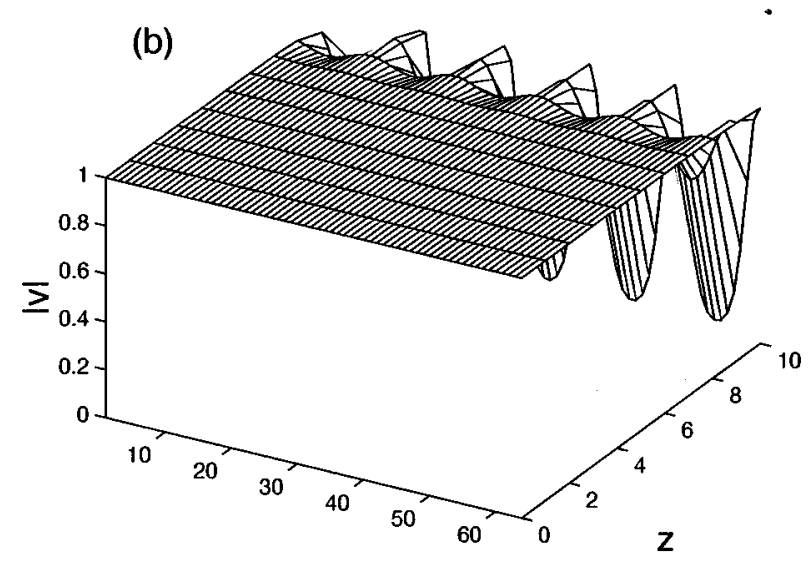

n

FIG. 6. Down-conversion with plane waves. Boundary conditions are periodic. Parameters are $\alpha=-2.9, \eta=0.5$. Input: exp $\left(-i k_{3} n\right)$ in $V, 0.0002 \exp \left(-i k_{1} n\right)$ in $W, k_{3}=-0.25, k_{1}=0.15$. (a) Fundamental. (b) Second harmonic. each other as they propagate along $z$. This walkoff will counteract the efficiency of the down-conversion and will eventually delocalize the beams. However, in Fig. 7 the initial stage of the down-conversion is clearly seen. Complete depletion of the $k_{3}$ component in $V$ is nearly obtained at $z$ $\approx 25$. At this point there is a mixture between an interference

(a)

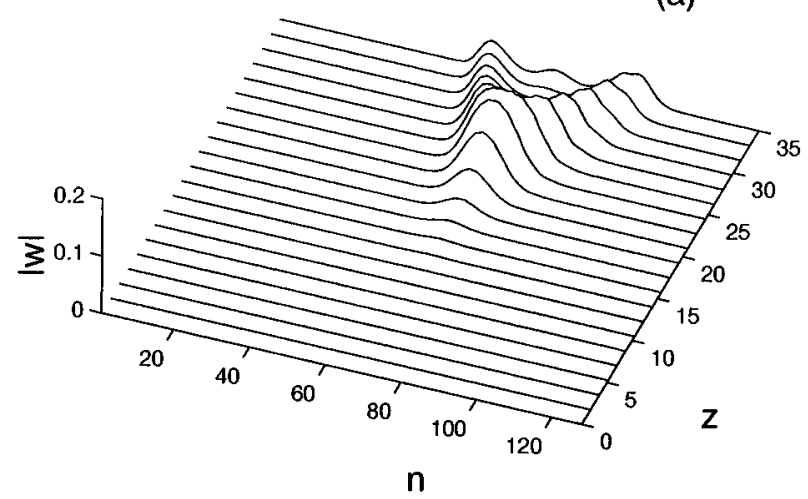

(b)

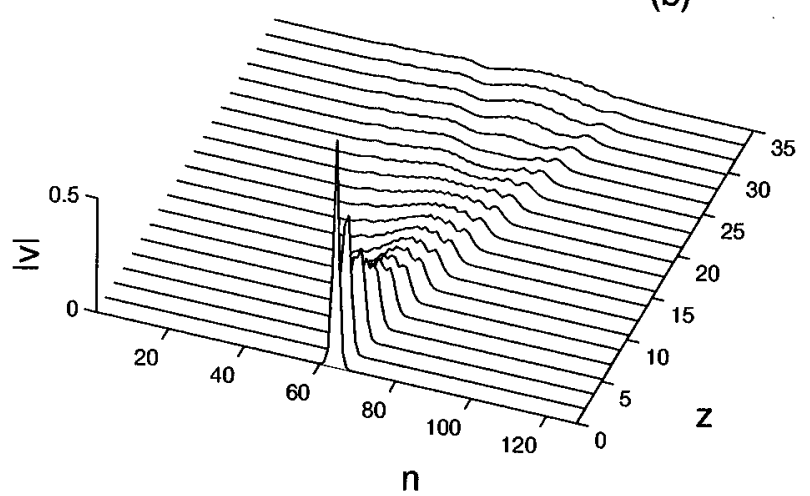

FIG. 7. Down-conversion with beams. Boundary conditions are open ends. Parameters are $\alpha=-2.9, \quad \eta=0.5$. Input: $f(n) \exp$ $\left(-i k_{3} n\right)$ in $V, 0.0002 f(n) \exp \left(-i k_{2} n\right)$ in $W, \quad k_{3}=-0.25, \quad k_{2}$ $=-0.4$, and $f(n)=\operatorname{sech}\left(n-n_{0} / 2\right)$. (a) Fundamental. (b) Second harmonic. 

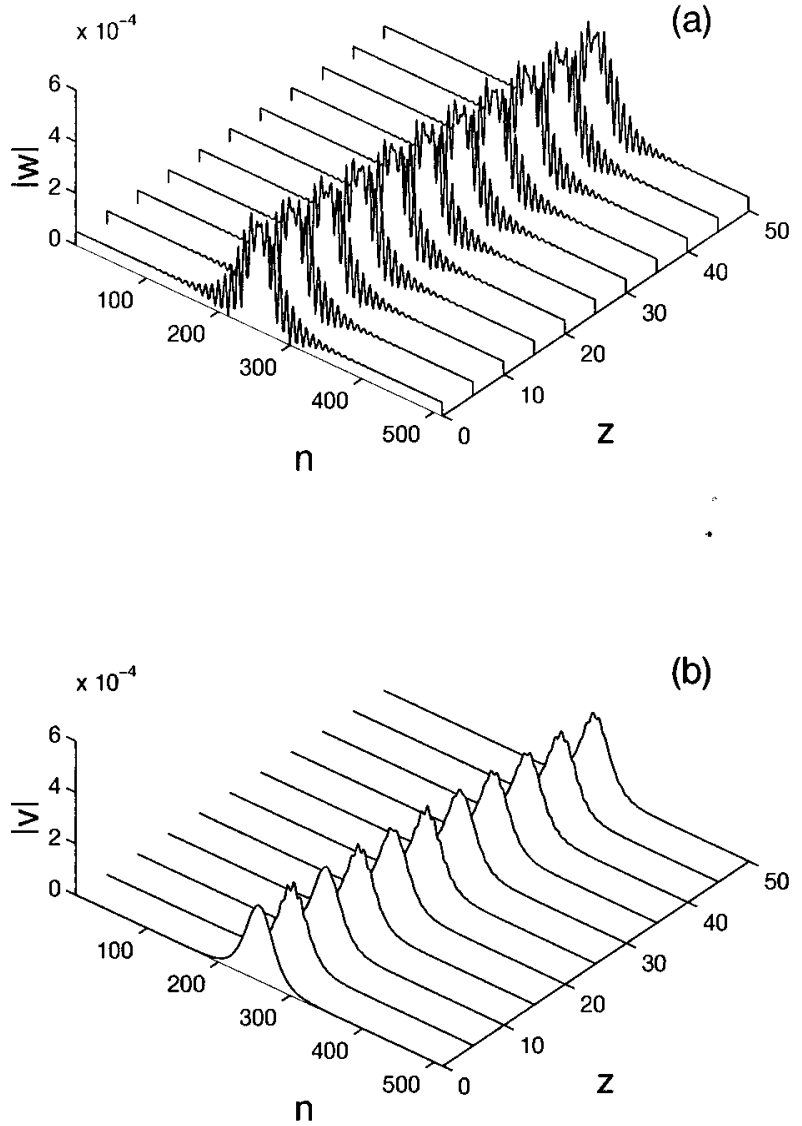

FIG. 8. Stable propagation of localized excitation. Boundary conditions are periodic. Parameters are $\eta=1.0, \alpha=-1.8$. Plane waves: $k_{1}=-0.5, k_{2}=0.2$. The velocity of the excitation is measured to be -0.79 . (a) Fundamental. (b) Second harmonic.

pattern from $k_{1}$ and $k_{2}$ in $|W|$ and walkoff. With $z>25$, the walkoff effects set in and the $k_{1}$ and $k_{2}$ components in $W$ walk away from each other with their respective velocities.

Although the beams in Fig. 7 remain localized, they are not stationary, since down-conversion is taking place. The stable solution given in Eq. (9) is based on a beam configuration where the three modes in the beams are stationary. In that configuration a stable balance exist in the intricate interplay between walkoff, harmonic energy exchange, and nonlinear phase changes. In Fig. 8 we show the stable propagation of such an excitation. The wave numbers are $k_{1}$ $=-0.5$ and $k_{2}=0.2$, yielding $\mathrm{v}_{1}=0.96, \mathrm{v}_{2}=-0.40$, and $v_{3}=0.59$. The value of $\vee$ was chosen to be 0.8 in the initial condition, which is reproduced by the simulation. The small wiggles generated on top of $|V|$ as the beam propagates along $z$ are caused by the formation of the higher order mixing terms $B_{1}$ and $B_{2}$. However, the excitation remains connected and propagates in a stable manner. Since $A_{2}$ in solution (9) has an infinite extension, we had to impose periodic boundary conditions in the simulations. Furthermore, numerical simulations confirm that the solution indicated in Eq. (10) is unstable. It is clear from the decay instability in Fig. 6 that solution (10), which has a constant background in $A_{3}$, will also be modulationally unstable.

\section{CONCLUSIONS}

In summary, we have shown that both efficient two-wave and three-wave interaction takes place in a lattice with quadratic nonlinearity when the interacting waves are tilted to a proper angle with respect to each other.

In the degenerate case with only two interacting waves, we focused on energy conversion from the fundamental to the second harmonic and detected the bandwidth of this process in terms of the sensitivity to the phase tilt (transverse wavenumber) of the incoming fundamental.

With three interacting waves the dynamics are potentially richer. We showed the existence of decay instability in the lattice that couples energy from $k_{3}$ to $k_{1}$ and $k_{2}$ and stable propagation of a localized excitation. It is worth noting that the three-wave components have different transverse wave numbers and therefore different propagation angles in free space. Thus, when energy is transferred from, say, $k_{2}$ to $k_{1}$ as in Fig. 7, the direction of light will have changed at the output of the waveguides according to the phase tilt $k_{1}$ $-k_{2}$. The direction of propagation is determined by the angle $\phi \approx k / Q$, where $Q$ is the wave number of the relevant carrier wave (see Fig. 1). Assuming the wavelength of the fundamental to be $\lambda \sim 1.5 \mu \mathrm{m}$ and the waveguide separation to be $\sim 10 \mu \mathrm{m}$, one finds that the change in angle is $\sim 0.75^{\circ}$ for the simulation shown in Fig. 7. The change in angle will be accompanied by a change in wavelength according to $q$, which depends on the scaling of $z$ and thus on the actual nearest neighbor coupling strength between the waveguides.

The results show the general nature of parametric wave interaction that may come into view in any lattice exhibiting a quadratic nonlinearity.

\section{ACKNOWLEDGMENTS}

The work has been supported by the bilateral agreement JNICT/The Danish Research Academy under Grant No. 1996-125-77. M.D.C. and V.V.K. also acknowledge partial financial support from FEDER and Program PRAXIS XXI under Grant No. PRAXIS/2/2.1/FIS/176/94.
[1] D. J. Kaup, A. Reiman, and A. Bers, Rev. Mod. Phys. 51, 275 (1979).

[2] G. I. Stegeman, D. J. Hagan, and L. Torner, Opt. Quantum Electron. 28, 1691 (1996).

[3] V. M. Agranovich and A. M. Kamchatnov, Pis'ma Zh. Eksp.
Teor. Fiz. 59, 397 (1994) [JETP Lett. 59, 424 (1994)].

[4] V. M. Agranovich, O. A. Dubovsky, and A. M. Kamchatnov, J. Phys. Chem. 98, 13607 (1994); Chem. Phys. 198, 245 (1995).

[5] S. A. Dubovskii and A. V. Orlov, Fiz. Tverd. Tela 38, 1221 
(1996) [Phys. Solid State 38, 675 (1996)]; 38, 1931 (1996) [38, 1067 (1996)].

[6] O. Bang, P. L. Christiansen, and C. Balslev Clausen, Phys. Rev. E 56, 7257 (1997).

[7] T. Peschel, U. Peschel, and F. Lederer, Phys. Rev. E 57, 1127 (1998).

[8] S. Darmanyan, A. Kobyakov, and F. Lederer, Phys. Rev. E 57, 2344 (1998).

[9] S. Darmanyan, A. Kamchatnov, and F. Lederer, Phys. Rev. E 58, R4120 (1998).

[10] P. D. Miller and O. Bang, Phys. Rev. E 57, 6038 (1998).

[11] S. P. Novikov, S. V. Manakov, L. P. Pitaevskii, and V. E. Zakharov, Theory of Solitons. The Inverse Scattering Method
(Plenum, New York, 1984); M. J. Ablowitz and H. Segur, Solitons and the Inverse Scattering Transform (SIAM, Philadelphia, 1981).

[12] Similar dynamical phenomena in one-dimensional discrete systems have been described in V. V. Konotop, Phys. Rev. E 54, 4266 (1996); Phys. Rev. B 55, R11 926 (1997).

[13] A. P. Sukhorukov, Nonlinear Wave Interactions in Optics and Radiophysics (Nauka, Moscow, 1988) (in Russian).

[14] This assumption is not crucial for our theory. The same evolution equation can be obtained in a finite lattice with periodic boundary conditions [V. V. Konotop, Phys. Rev. E 53, 2843 (1996)]. 